Sonderdruck aus

Hoppe-Seyler's Zeitschrift für Physiologische Chemie

Walter de Gruyter \& Co., Berlin 30

Hoppe-Seyler's Z. Physiol. Chem.

Bd. 353, S. 1633-1637, Oktober 1972

\title{
Isolierung eines Trypsin-ähnlichen Enzyms (Akrosin) aus Eberspermien
}

\author{
Edwin Fink, Hans Schießler, Marianne Arnhold und Hans Fritz*
}

(Der Schriftleitung zugegangen am 17. August 1972)

Herrn Professor Dr. Dr. Eugen Werle zum 70. Geburtstag gewidmet

\begin{abstract}
Zusammenfassung: Aus Eberspermien wurde das Trypsin-ähnliche Enzym Akrosin isoliert durch a) Extraktion mit Essigsäure $(2 \%, \mathrm{~V} / \mathrm{V})$-Salzsäurelösung, $\mathrm{pH} \mathrm{1,8,} \mathrm{b)} \mathrm{Fraktionierung} \mathrm{über} \mathrm{Sephadex}$ G-75 in saurer Lösung und c) Affinitätschromatographie unter Verwendung wasserunlöslicher Bowman-Birk-Sojabohnen-Trypsininhibitor-Cellulose.
\end{abstract}

Das mit einer spezifischen Aktivität von $5 \mathrm{U}$ pro mg (Substrat: $N^{\alpha}$-Benzoyl-DL-arginin-p-nitroanilid) erhaltene Akrosin ist stabil in schwach saurer Lösung. Es wird aus neutralen bis schwach alkalischen Lösungen von Oberflächen, insbesondere Glas, stark adsorbiert.

\section{Isolation of a trypsin-like enzyme (acrosin) from boar spermatozoa}

Summary: The trypsin-like enzyme acrosin was isolated a) by extraction of washed boar spermatozoa with acetic acid $(2 \%, \mathrm{~V} / \mathrm{V})$-hydrochloric acid solution $\mathrm{pH} 1.8$, b) by fractionation on Sephadex G-75 in acidic solution and c) by affinity chromatography using water insoluble Bowman-

Aus gewaschenen Spermien lassen sich Extrakte mit einer relativ hohen Tosylarginin-methylesterspaltenden Aktivität gewinnen[1], die auf das Vorhandensein eines Trypsin-ähnlichen Enzyms (Akrosin) in den Akrosomenkappen der Spermien ${ }^{[2,3]}$ zurückzuführen ist. Mehrere Autoren haben inzwischen die Eigenschaften der Akrosine verschiedener Spezies unter Verwendung akrosomaler Extrakte untersucht ${ }^{[4-9]}$. Eine weitergehende Reinigung des

Abkürzungen: s. zu Beginn des method. Teils.

* Adresse: Priv.-Doz. Dr. H. Fritz und Dipl.-Chem. H. Schießler, Institut für Klinische Chemie und Klinische Biochemie der Universität München, D-8 München 2, Nußbaumstraße 20.

1 Waldschmidt, M., Hoffmann, B. \& Karg, H. (1966) Zuchthygiene 1, 15-21.

2 Stambaugh, R. \& Buckley, J. (1969) J. Reprod. Fert. 19, $423-432$.

3 Stambaugh, R. \& Buckley, J. (1970) Biol. Reprod. 3, 275- 282 .

4 Zaneveld, L. J. D., Polakoski, K. L., Robertson, R. T. \& Williams, W. L. (1971) in Proc. Int. Res. Conf. on
Birk soybean trypsin inhibitor cellulose. The acrosin obtained with a specific activity of $5 \mathrm{U}$ per $\mathrm{mg}$ (substrate: $N^{\alpha}$-benzoyl-DL-arginine-p-nitroanilide)

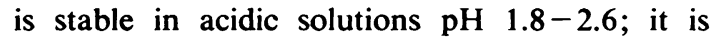
strongly adsorbed on surfaces, especially on glass, of neutral or weakly basic solutions.

Akrosins aus Kaninchenspermien beschrieben kürzlich Polakoski, Zaneveld und Williams ${ }^{[10]}$, sowie für das Akrosin aus Humanspermien Zaneveld, Dragoje und Schumacher[11]. Mit Hilfe der Affinitätschromatographie gelang uns die Herstellung von Akrosinpräparaten aus Eberspermien mit hoher spezifischer Aktivität.

Proteinase Inhibitors (Fritz, H. \& Tschesche, H., Hrsg.) S. 236-244, Walter de Gruyter, Berlin.

${ }^{5}$ Polakoski, K. L., Williams, W. L. \& McRorie, R. A. (1972) Fed. Proc. 31, 278.

6 Stambaugh, R. \& Buckley, J. (1971) Fed. Proc. 30, 1184.

7 Multamäki, S. \& Niemi, M. (1972) Int. J. Fertil. 17, $43-52$.

${ }^{8}$ Garner, D. L., Salisbury, G. W. \& Graves, Ch. N. (1971) Biol. Reprod. 4, 93-100.

${ }^{9}$ Ho, J. J. L. \& Meizel, S. (1970) J. Reprod. Fert. 23, 177- 179 .

10 Polakoski, K. L., Zaneveld, L. J. D. \& Williams, W. L. (1972) Biol. Reprod. 6, 23-29.

11 Zaneveld, L. J. D., Dragoje, B. M. \& Schumacher, G. F. B. (1972) Science, 177, 702-703. 


\section{Methodik und Ergebnisse}

Bestimmung der Akrosinaktivität: Als Substrat diente $N^{\alpha}$-Benzoyl-DL-arginin-p-nitroanilid (BAPA). Zur Methodik s. 1. c. ${ }^{[12]}$. Die Meßbedingungen bei Verwendung der Substrate $\boldsymbol{N}^{\alpha}$-Benzoyl-L-lysin-p-nitroanilid (BLNA), $N^{\alpha}$-Benzoyl-L-arginin-äthylester (BAEE) in Kombination mit Alkohol-Dehydrogenase (ADH) und Azokasein sind ebenfalls in 1. c. ${ }^{[12]}$ angegeben.

Proteinbestimmung: Die Proteinbestimmung erfolgte mit der Biuret-Methode oder durch Extinktionsmessung bei $278 \mathrm{~nm}$, wobei eine Human- $\gamma-$ Globulinlösung (Behringwerke AG, Marburg) bekannter Konzentration $\left(E_{280}^{1 \%}=11,8\right)$ als Eichstandard verwendet wurde.

Gewinnung der Spermienextrakte: Ebersperma wurde unter Zusatz von Konservierungsmitteln in der Prüfund Besamungsstation München-Aubing e. V.* gesammelt. Der im Laufe von jeweils ca. 10 Tagen anfallende Spermapool (ca. $6 \mathrm{l}$ ) wurde $90 \mathrm{~min}$ lang bei $4^{\circ} \mathrm{C}$ und $1000 \times g$ zentrifugiert. Das Sediment wurde insgesamt dreimal in jeweils $60 \mathrm{ml}$ physiologischer Kochsalzlösung suspendiert und anschließend zentrifugiert. Das so erhaltene Spermiensediment wurde in $60 \mathrm{~m} / 2$ proz. Essigsäure, die durch Zugabe von $2 \mathrm{~N} \mathrm{HCl}$ auf $\mathrm{pH}$ 1,8 eingestellt worden war, 5 min suspendiert. Nach 10 min langer Zentrifugation bei $1000 \times g\left(4^{\circ} \mathrm{C}\right)$ wurde der milchig trübe Uberstand abdekantiert und $100 \mathrm{~min}$ lang bei $17000 \times g$ zentrifugiert. Der klare Überstand wurde durch Ultrafiltration (Amicon-Zelle, UM-2 Membran, $4^{\circ} \mathrm{C}$ ) auf $1 / 4$ des Ausgangsvolumens eingeengt. Die spezifische Aktivität dieser essigsauren Spermienextrakte lag bei 0,05-0,08 U (BAPA)/mg Biuret-Protein (vgl. dazu die Diskussion).

Zur quantitativen Auswertung wurden $130 \mathrm{~m} l$ frisch ejakuliertes Ebersperma (pH 7,6, 122 Mill. Spermien/ $\mathrm{ml}$ ) wie beschrieben aufgearbeitet: Zentrifugation für $60 \mathrm{~min}$ bei $1000 \times g$, 3maliges Auswaschen des Sediments mit je $30 \mathrm{ml}$ physiol. $\mathrm{NaCl}$-Lösung, 5malige Extraktion des Spermiensediments mit je $10 \mathrm{ml}$ der Essigsäure-Salzsäure-Lösung vom pH 1,8. Nach Konzentrierung der sauren Extrakte auf $10 \mathrm{~m} /$ durch Ultrafiltration erfolgte, wie nachstehend beschrieben, die Fraktionierung an Sephadex G-75. Die dabei eluierte Akrosinfraktion (vgl. die Abbildung) enthielt eine $\mathrm{Ge}$ samtaktivität von 11 U (BAPA). Aus $10^{8}$ Spermien wurden demnach $70 \mathrm{mU}$ (BAPA) Akrosin gewonnen.

Fraktionierung an Sephadex G-75: Entsprechend den unterschiedlichen Molekülgrößen wird bei der Fraktionierung der konzentrierten sauren Spermienextrakte über eine Sephadex-G-75-Säule die Akrosinfraktion

* Herrn H. Kreutmayer, Herrn G. Obernöder und Herrn $F$. Schrödobler möchten wir für ihre Unterstützung bei der Sammelaktion bestens danken

12 Schießler, H., Fritz, H., Arnhold, M., Fink, E. \& Tschesche, H. (1972) diese Z. 353, 1638-1645, nachstehend. vor der Inhibitorfraktion [13] eluiert. Unter geeigneten Bedingungen ist eine vollständige Trennung beider Fraktionen zu erreichen (s. Abbildung). Auf die Säule aufgetragen wurden jeweils $7-10 \mathrm{~m} l$ Konzentrat; in den akrosinhaltigen Eluaten wurde eine Gesamtaktivität von 20-31 U (BAPA) gefunden. Die Inhibitorfraktion enthielt durchschnittlich 5-8 IU für Trypsin.

Für die spezifischen Spaltungsaktivitäten in den eluierten Akrosinfraktionen wurden Werte zwischen 1 und $1,2 \mathrm{U}$ (BAPA)/mg Protein gemessen. Dies entspricht einer 12-18fachen Anreicherung bei diesem Isolierungsschritt.

Die vereinigten akrosinhaltigen Fraktionen wurden durch Zugabe von $2 \mathrm{~N} \mathrm{NaOH}$ auf $\mathrm{pH} 7,8$ eingestellt. Der dabei in wechselnden Mengen ausfallende ProteinNiederschlag (bis zu $20 \%$ des Proteingehalts der Akrosinlösung) enthielt nur wenig Akrosinaktivität; er wurde abzentrifugiert und der klare Überstand für den nachfolgenden Reinigungsschritt oder für Testzwecke (,Akrosin vor der Affinitätschromatographie“, s. auch l. c. $\left.{ }^{[14]}\right)$ verwendet.

Affinitätschromatographie: In dem verwendeten wasserunlöslichen Inhibitor-Cellulosederivat der Fa. E. Merck Darmstadt* ist der Bowman-Birk-Trypsininhibitor aus Sojabohnen über einen Spacer an Aminoalkylcellulose fixiert. Das Inhibitor-Cellulosederivat wurde zur Solubilisierung evtl. löslicher Anteile ausgiebig mit $2 \mathrm{M} \mathrm{NaCl}$, 5proz. Caseinlösung, 4M Harnstoff und dem Äquilibrierpuffer $(0,1 \mathrm{M}$ Triäthanolamin/ $/ \mathrm{HCl}, 0,4 \mathrm{M} \mathrm{NaCl}$, $\mathrm{pH} 7,8$ ) gewaschen. Alle Operationen wurden bei $0-4^{\circ} \mathrm{C}$ durchgeführt.

Das mit dem zuletzt genannten Puffer äquilibrierte Inhibitor-Cellulosederivat wurde $\mathbf{3 0} \mathrm{min}$ in der Akrosinlösung suspendiert. Danach wurde zentrifugiert und das Celluloseadsorbat $5 \mathrm{mal}$ mit je $50 \mathrm{ml}$ Äquilibrierpuffer ausgewaschen (suspendieren, zentrifugieren). Die Ablösung des Akrosins erfolgte durch zweimaliges Suspendieren (je $10 \mathrm{~min}$ ) des Adsorbates in $10-20 \mathrm{ml}$ $0,4 \mathrm{M} \mathrm{KCl} / \mathrm{HCl}, \mathrm{pH} 1,8$ oder 2proz. Essigsäure (plus 2N $\mathrm{HCl}$ ), pH 1,8. Die Ergebnisse sind in der Tabelle zusammengefaßt. Die Beladungskapazität der eingesetzten Inhibitor-Cellulosemengen war in keinem Falle erschöpft.

Die Bestimmung der spezifischen Spaltungsaktivitäten der sauren Akrosineluate ergab Werte um 5 U (BAPA)/ mg Protein, was einer 3-4fachen Anreicherung bei diesem Reinigungsschritt entspricht.

* Herrn Dr. H. Lang und Herrn Dr. N. Hennrich, E. Merck Darmstadt, sind wir für die Herstellung und Überlassung des Inhibitor-Cellulosederivates sehr dankbar.

13 Das Molekulargewicht des Inhibitors liegt bei 13000 , s. Fink, E., Klein, G., Hammer, F., Müller-Bardorff, G. \& Fritz, H. (1971) in 1. c. ${ }^{[4]}$, S. 225-235.

14 Fritz, H., Förg-Brey, B. Schießler, H. Arnhold, M. \& Fink, E. (1972) diese Z. 353, 1010-1012. 


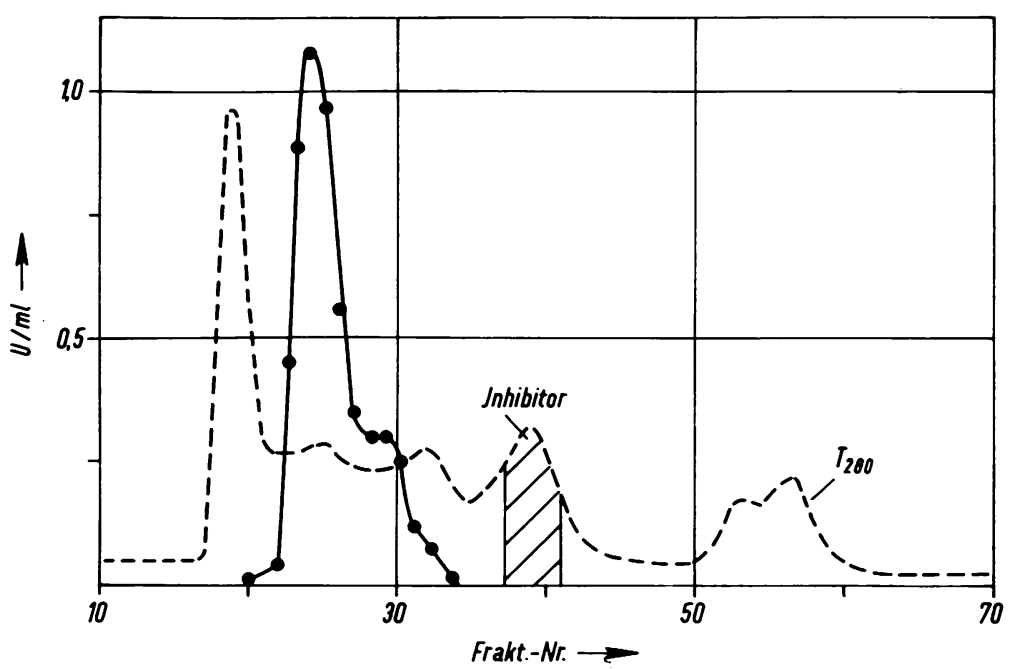

Abbildung. Fraktionierung der Spermienextrakte an Sephadex G-75 in saurer Lösung.

Bedingungen : $125 \times 2,8-\mathrm{cm}-S a ̈ u l e ~(S e p h a d e x ~ G-75)$, äquilibriert und entwickelt mit 2proz. Essigsäure plus $2 \mathrm{~N} \mathrm{HCl}$ bis $\mathrm{pH} 1,8 \mathrm{mit}$ einer Geschwindigkeit von $30 \mathrm{ml} / \mathrm{h}$ und $10 \mathrm{ml} /$ Fraktion.

Ordinate: Akrosinaktivität in U (BAPA) $/ \mathrm{ml} . T_{280}=$ Transmission bei $280 \mathrm{~nm}$.

Molekulargewichtsabschätzung: Die größenordnungsmäßige Bestimmung des Molekulargewichts durch Gelfiltration ergab unter den in der Abbildung angegebenen Bedingungen einen Wert um 56000. Als Bezugssubstanzen dienten Dextranblau, Chymotrypsinogen und Ribonuclease.

\section{Diskussion}

1. Herstellung akrosomaler Extrakte: Zur Herstellung akrosomaler Extrakte von Spermien werden bevorzugt detergentienhaltige, schwach basische (pH 7,5) Pufferlösungen verwendet ${ }^{[2-4,10,11,15-18]}$. Aus ejakulierten Spermien erhält man dabei Extrakte, in denen freies Akrosin nicht oder nur in sehr geringer Aktivität nachzuweisen ist ${ }^{[4,15,16]}$, da es maskiert in an Inhibitoren gebundener Form vorliegt. Zaneveld, Polakoski und Williams haben dies neuerdings in überzeugender Weise demonstriert

15 Polakoski, K. L., Zaneveld, L. J. D. \& Williams, W. L. (1972) Biochem. Biophys. Res. Commun., im Druck.

16 Zaneveld, L. J. D., Polakoski, K. L. \& Williams. W. L. (1972) Biol. Reprod., im Druck.

17 Allison, A. C. \& Hartree, E. F. (1970) J. Reprod. Fert. 21, 501-515.

18 Teichman, R. J. \& Bernstein, M. H. (1971) J. Reprod. Fert. 27, 243-248.
[15,16]. Die zur Isolierung des Akrosins notwendige Trennung von Akrosin und Inhibitor gelingt durch Fraktionierung dieser Extrakte über Molekularsiebe in saurer Lösung ${ }^{[11]}$, anscheinend aber auch durch Ionenaustauschchromatographie ${ }^{[10]}$.

Vorversuche zeigten, daß die Extraktion ejakulierter Eberspermien mit 2proz. Essigsäure (plus $2 \mathrm{~N}$ $\mathrm{HCl}$ ) vom $\mathrm{pH} 2,6(-1,8)$ um etwa $30-50 \%$ höhere Akrosinausbeuten ergibt als die Verwendung der detergentienhaltigen Pufferlösungen. Mit der sauren Lösung sind 3 Extraktionen (je ca. 15 min langes Stehenlassen bei Zimmertemperatur) ausreichend, während nach viermaliger Extraktion von Spermien mit der Detergentienlösung (Hyamin plus Triton $\mathrm{X}-100$, je $1 \mathrm{~h}$ bei $37^{\circ} \mathrm{C}$ ) anschließend mit der Essigsäurelösung noch weiteres Akrosin ablösbar ist. Offensichtlich wird die Akrosomenmembran, wie für lysosomale Membranen bekannt, in der sauren Lösung besonders rasch und vollständig durchlässig bzw. das Akrosin - sofern es an die Membran fixiert ist - vollständig abgelöst. Dabei wirkt sich evtl. auch die sofortige Dissoziation des Akrosin-Inhibitor-Komplexes in der sauren Extraktionslösung günstig aus. Die skizzierten Ergebnisse stimmen insofern auch mit den Angaben von Allison und Hartree[17] überein, die fanden, da $\beta$ bei Detergentienbehandlung nur $50-60 \%$ der 
Akrosomenkappen von den Spermienköpfen abgelöst werden.

\section{Aktivitätsbestimmung in den akrosomalen Extrak-} ten: Die Akrosinaktivität akrosomaler Extrakte ist normalerweise erst nach Abtrennung des Inhibitors, also nach dem Gelfiltrationsschritt, exakt meßbar. Unter bestimmten Bedingungen, sofern z. B. kein Inhibitorüberschuß gegenüber dem Akrosin vorhanden ist, dürfte die Akrosinbestimmung auch in den sauren akrosomalen Extrakten bzw. in auf pH 1,8-3 angesäuerten Detergentienextrakten möglich sein. Wird nämlich eine entsprechende Verdünnung eines sauren (angesäuerten) Extraktes von gut gewaschenen Spermien (um überschüssigen Inhibitor zu entfernen!) direkt in die bereits alle Reagentien enthaltende Meßküvette pipettiert, so erhält man beim sehr empfindlichen BAEE/ADHSystem - wie auch beim direkten BAEE-Test ${ }^{[16]}$ - über einen genügend langen Zeitraum konstante $\Delta E /$ min-Werte. Infolge der extrem niedrigen Konzentrationen der Komplexpartner Akrosin und Inhibitor im Meßansatz (unter $3 \times 10^{-10} \mathrm{M}$ !) und des bei der $\mathrm{pH}$-Umstellung sofort wirksamen großen Substratüberschusses ist die Geschwindigkeit der Komplexbildung offensichtlich minimal und im verwendeten Testsystem deshalb nicht mehr nachweisbar. Beim BAPA-Test dagegen ist unter denselben Voraussetzungen die Komplexbildung in der laufenden Abnahme der Extinktionsänderung/Zeiteinheit zu erkennen. Zaneveld und Mitarb. ${ }^{[16]}$ diskutieren auch das Vorliegen verschiedener Inhibitorformen (nativ, modifiziert) mit unterschiedlicher Affinität zum Akrosin.

Aus den in dieser und der nachstehenden Arbeit ${ }^{[12]}$ angeführten Daten errechnen sich für den Akrosingehalt von $10^{8}$ Eberspermien folgende Aktivitäten: $70 \mathrm{mU}$ (BAPA) bzw. $5 \mathrm{mU}$ (BLNA) bzw. $800 \mathrm{mU}$ (BAEE/ADH). Die Akrosinmenge von $10^{8}$ Eberspermien spaltet Azokasein mit einer Wirksamkeit, die der von $1 \mu \mathrm{g}$ Trypsin (Novo Industri $\mathrm{A} / \mathrm{S}$, ca. $70 \%$ rein) entspricht.

3. Isolierung und Eigenschaften des Akrosins: Die im Elutionsdiagramm der Gelfiltration sichtbare Schulter im Akrosingipfel deutet darauf hin, daß das Akrosin überwiegend als dimeres Assoziat (Hauptfraktion) neben einer kleineren Menge Monomerem eluiert wird. Diese Annahme wird gestützt durch die Ergebnisse der Molekulargewichtsbestimmungen am Kaninchenakrosin[10]. Polakoski und Mitarb. fanden bei der Gelfiltration einen Wert um 55000, bei der Elektrophorese in Poly- acrylamid in Gegenwart von Natriumdodecylsulfat jedoch nur einen Wert von 27300.

Bei der Affinitätschromatographie wurde ein Überschuß an Inhibitoradsorbens eingesetzt, so daß die Daten in der Tabelle keinen Rückschluß auf die maximale Beladungskapazität der Inhibitor-Cellulose erlauben. Zur Ablösung des Akrosins vom Adsorbat wurde dieses nur zweimal mit der sauren Salz-Pufferlösung eluiert. Aus verdünnteren Lösungen (also bei der dritten und weiteren Elutionen) wird Akrosin von diesem Reinheitsgrad sehr effektiv an Oberflächen adsorbiert und geht somit verloren. Eine Verbesserungsmöglichkeit sehen wir hier in der Verwendung evt. neuer wasserunlöslicher Inhibitorderivate im Säulenverfahren sowie von akrosinstabilisierenden Zusätzen. Da das isolierte Akrosin u. U. ein Gemisch von Isoenzymen darstellt ${ }^{[8,9]}$, versuchen wir $\mathrm{z}$. Zt. durch Ionenaustauschchromatographie eine weitere Auftrennung und Reinigung des bei der Affinitätschromatographie anfallenden Akrosins zu erzielen, das nach unseren Berechnungen zu etwa $50 \%$ rein ist. In konzentrierterer Form ist dieses Akrosin im $\mathrm{pH}$ Bereich 1,8-2,6 bei Zimmertemperatur über Tage

Tabelle. Reinigung von Eber-Akrosin durch Affinitätschromatographie im Batchbetrieb.

Die experimentellen Bedingungen sind im Text beschrieben.

\begin{tabular}{lccl}
\hline $\begin{array}{l}\text { Eingesetzte } \\
\text { Inh.-Cellulose } \\
{[\mathrm{mg}]}\end{array}$ & $\begin{array}{c}\text { Akrosinmenge } \\
\text { eingesetzt } \\
{\left[\mathrm{U}^{\mathrm{b}}\right.}\end{array}$ & $\begin{array}{l}\text { gebunden } \\
{[\mathrm{U}]^{\mathrm{b}}}\end{array}$ & $\begin{array}{l}\text { abgelöst } \\
{[\%]}\end{array}$ \\
\hline 100 & 4 & 4 & 35 \\
& 3,8 & 3,8 & 79 \\
\hline 300 & 4 & 4 & 58 \\
& 4,4 & 4,4 & 63 \\
& 2,4 & 2,4 & 70 \\
& 15 & 14,4 & 57 \\
& 14 & 14 & 75 \\
& 6 & 6 & 60 \\
& 14,4 & 14,4 & 55 \\
\hline 400 & 15 & 15 & 65 \\
& 14 & 14 & 75 \\
& 6 & 6 & 58 \\
& 9,6 & 9,6 & 70 \\
\hline
\end{tabular}

a Bezogen auf die gebundene Menge.

b Substrat: BAPA.

19 Zaneveld, L. J. D., Polakoski, K. L. \& Williams. W. L. (1972) Biol. Reprod. 6, 30-39. 
und im Kühlschrank über Wochen stabil. Im Neutralbereich (pH 7-8) bleibt die Akrosinaktivität nach einem geringen initialen Abfall (5-15\%) ebenfalls einige Tage lang bei $0-4^{\circ} \mathrm{C}$ konstant. Die Autoproteolyse ist demnach beim Eberakrosin wesentlich weniger stark ausgeprägt als beim Kaninchenakrosin ${ }^{[19]}$. Der auffallend hohe Verlust (80\% und mehr) an Kaninchen-Akrosinaktivität bei der Umstellung saurer Lösungen auf pH-Werte über 7 dürfte demnach überwiegend auf Inakti- vierungseffekte durch Adsorption an Glasoberflächen zurückzuführen sein.

Weitere enzymatische Eigenschaften des Eberakroins sind in der nachfolgenden Arbeit ${ }^{[12]}$ beschrieben.

Mit Mitteln des Sonderforschungsbereiches 51, München, finanziert. Unser Dank gilt auch Herrn Prof. Dr. Dr. E. Werle für die Unterstützung dieser Arbeiten und Herrn Doz. Dr. H. Tschesche für den zur Verfügung gestellten Arbeitsplatz (für Herrn H. Schießler). 